Deif and ElMaraghy. Published in the Journal of Production, Planning \& Control 20(8):737-749. October 2010.

\title{
Modelling and analysis of dynamic capacity complexity in multi-stage production
}

\author{
Ahmed M. Deif a and Hoda A. ElMaraghy ${ }^{\text {b* }}$ \\ ${ }^{a}$ Department of Industrial Systems, University of Regina, Canada; ${ }^{b}$ Department of Industrial and Manufacturing \\ Systems Engineering, University of Windsor, Room 204, Odette Building, 401 Sunset Avenue, Canada
}

\begin{abstract}
The uncertainty associated with managing dynamic capacity problem is the main source of its complexity. This article presents a system dynamics approach to model and analyse operational complexity of dynamic capacity in multi-stage production. The unique feature of this approach is that it captures the stochastic nature of three main sources of complexity associated with dynamic capacity. These are the demand, internal manufacturing delay and capacity scalability delay. The developed model was demonstrated by an industrial case study of multi-stage printed circuit board assembly line. The analysis of simulation experiments showed that ignoring complexity sources can lead to wrong decisions concerning both scaling levels and backlog management decisions. In addition, a general trade-off between the controllability and complexity of the dynamic capacity was illustrated. Finally, comparative analysis of the effect of each of these sources on the complexity level revealed that internal delay has the highest impact on dynamic capacity efficiency. Guidelines and recommendations for better capacity management and reduction of its complexity are presented.
\end{abstract}

Keywords: complexity; system dynamics; capacity management

\section{Introduction}

The typical problem in capacity planning is to decide on the time, the amount of investment and the resources (equipment, facilities, systems and people) to use in a manufacturing site at any time. Extensive research has been conducted to study the optimal capacity planning under different conditions (see Manne (1967), Luss (1982) and van Mieghem (2003) for extensive review on optimal capacity planning). The inherent complexity within the capacity planning process is one of the parameters that has a significant influence on the capacity management decisions and yet has received little attention to date. Discussing the complexity of capacity planning requires positioning the capacity planning problem within the proper framework and determining the type(s) and sources of complexity present in this domain.

In today's competitive market, manufacturing enterprises face the challenge of being responsive to changeable market demand while keeping a cost effective level of production. Facing such a challenge would have been very difficult without the new manufacturing paradigms and the technological enablers to allow changing their functionality as well as their capacity (Wiendahl et al. 2007). Such dynamic market environment with the continuous advancement of technology makes the management of the capacity change and reconfiguration very dynamic (Deif and ElMaraghy 2006). Therefore, capacity planning is inherently a dynamic problem.

Complexity covers a broad scope and is associated with systems that are difficult to understand, describe, predict or control. Complexity can be generally classified into structural and operational complexity. Structural complexity refers to the static design dimension of the system (Deshmukh et al. 1998) and the different system components and how they relate to each other (ElMaraghy et al. 2005, ElMaraghy 2006). Operational complexity, on the other hand, is defined as the uncertainty associated with the dynamic system operation (Frizelle 1998).

Since the capacity planning problem is dynamic in nature with various uncertainties, it can be classified as a type of operational complexity. However, since there are various definitions of operational complexity, it is important to clearly define operational complexity as used in this research. Operational complexity is defined as the effort expressed in terms of the magnitude and frequency of dynamic capacity planning to determine when and by how much the capacity should be scaled in response to demand due to internal and external sources of uncertainty.

In this article, the operational complexity of the dynamic capacity planning problem is investigated.

\footnotetext{
*Corresponding author. Email: hae@uwindsor.ca
} 
The analysis and identification of significant factors affecting the complexity of this capacity planning problem is of particular interest. The study will involve first developing a dynamic model for capacity scalability in multi-stage production (as a general representation of various manufacturing activities) using the system dynamics (SD) approach. The model incorporates three main sources of uncertainty in the capacity scaling process. The demand as the first source is modelled as a stochastic input with 'pink noise' to reflect practical market fluctuation. The second major source is the internal manufacturing delay at each stage, which is modelled as a stochastic time parameter depending on the production nature of each stage. The last source is the capacity scalability delay, which is modelled as a varying time function based on the type of the capacity unit to be scaled up or down at each stage. Various analyses to study the impact of each of these uncertainty sources on the complexity of the dynamic capacity planning are conducted.

\section{Literature review}

In this section, the two major dynamic methodologies that were used to handle the dynamic capacity planning are reviewed. The first methodology is the control-theoretic approach (mainly feedback control), which was used to model and control the capacity scaling process in different systems. The second methodology is SD introduced by Forrester (1961) and aims at understanding how the physical process, the information and the managerial policies of capacity scalability interact together. In a general sense, the control-theoretic approaches are more popular and better developed for handling the capacity scaling from a control perspective, while SD approaches are more suited for comparing and assessing different capacity scalability policies.

A dynamic model developed by Duffie and Falu (2002) for closed loop production planning and control (PPC) was proposed to control work in process (WIP) and capacity using control-theoretic approaches. They investigated the effect of choosing different capacity scaling controller gains as well as the WIP controller gains on system performance and how this can be used to achieve required system responses. Kim and Duffie (2004) extended this work to study the effect of capacity disturbances and capacity delays on system performance in single workstations and further applied it to multiple workstations in Kim and Duffie (2005). Their results highlighted the fact that if capacity can be adjusted more often with less delay, the system's performance would be highly improved in changing demand environments.

Another dynamic model that manipulates feedback control with the help of logistics operating curves, developed by Nyhuis (1994) to control WIP and capacity of manufacturing systems, was presented by Wiendahl and Breithaupt (1999, 2000). In this approach, the required capacity scalability was found using flexibility curves, which indicate the time delay of each capacity scaling step. The capacity scaling controller chooses the best capacity scaling decision based on balancing the backlog value and acceptable delay.

Asl and Ulsoy (2002) presented a dynamic approach to capacity scalability modelling in reconfigurable manufacturing systems (RMS) based on the use of feedback control. Sub-optimal solutions that are robust against demand variations and partially minimise the cost of capacity scalability were presented.

Deif and ElMaraghy (2006) developed a dynamic model for capacity scaling in RMS and analysed the model based on control-theoretic approaches to indicate the best design for the scaling controller. The results highlighted the importance of accounting for the different physical and logical delays together with the trade-off decisions between responsiveness and cost when designing the capacity scaling controllers. They further introduced an optimisation unit to the capacity scalability model to optimally decide on the exact value of the scaling controller gain (Deif and ElMaraghy 2007a).

Wikner et al. (2007) modified the famous automatic pipeline inventory and order-based production control system (APIOBPCS) used for make-to-stock to deal with make-to-order systems using the dynamic surplus capacity. They showed that these systems can maintain agility and decrease the backlog levels by introducing a controller to account for the backlog resulting from the capacity scaling delays while responding to changing demands. The controller gain value was function of both the manufacturing lead-time and the demand.

Examples of manipulating SD models to tackle the capacity planning needs include an attempt by Evans and Naim (1994) that aimed at developing an SD model for supply chains with capacity constraints and studying the effect of capacity constraints on the system's performance and overall cost.

Helo (2000) suggested a capacity-based supply chain model that includes a mechanism for handling the trade-off between lead-time and capacity utilisation. It was shown that this capacity analysis (including surge effects) in supply chains would improve their responsiveness. 
Goncalves et al. (2005) highlighted the issue of capacity variation in their push-pull manufacturing SD model through the effect of capacity utilisation on the production start rate. They also showed how the sales and production effects interact to destabilise the system and degrade its performance.

Anderson et al. (2005) considered logical capacity scalability in supply chains for service and custom manufacturing. They showed the effect of reducing lead-time and sharing the demand information on improving the system performance. In addition they proposed some polices to handle and reduce backlog in these systems.

Vlachos et al. (2007b) proposed a model to study the long-term behaviour of reverse supply chains applied to remanufacturing. For that purpose, they examined efficient remanufacturing and collection capacity expansion policies that maintain profit while considering direct and indirect factors.

Deif and ElMaraghy (2007b) proposed an SD single stage model for capacity scalability in maketo-order manufacturing. They used various performance measures to examine the best scaling policy under different demand scenarios. They showed that the best scalability policy would be based on both the marketing strategy and the operational production objectives. This article builds over these findings and adopts that hybrid scaling policy. In addition, it extends the analysis into a multi-stage manufacturing environment.

The previous dynamic approaches to model and analyse the dynamic capacity planning problem focused on either controlling the capacity scalability process or exploring different policies to hedge against various internal and external disturbances. Although they offered good solutions for both problems, no work has been reported to study the operational complexity associated with this problem. Thus the work presented in this article is motivated by the need to better understand the sources of operational complexity and their degree of influence in dynamic capacity planning. It is believed that such understanding would result in reducing the complexity in the dynamic capacity planning problem and generally leads to better management of dynamic capacity in manufacturing systems in an increasingly changeable environment.

\section{Stochastic dynamic capacity model in multi-stage production}

A stochastic dynamic model for the capacity problem with its different sources of complexity has been formulated. Figure 1 contains a dynamic model for

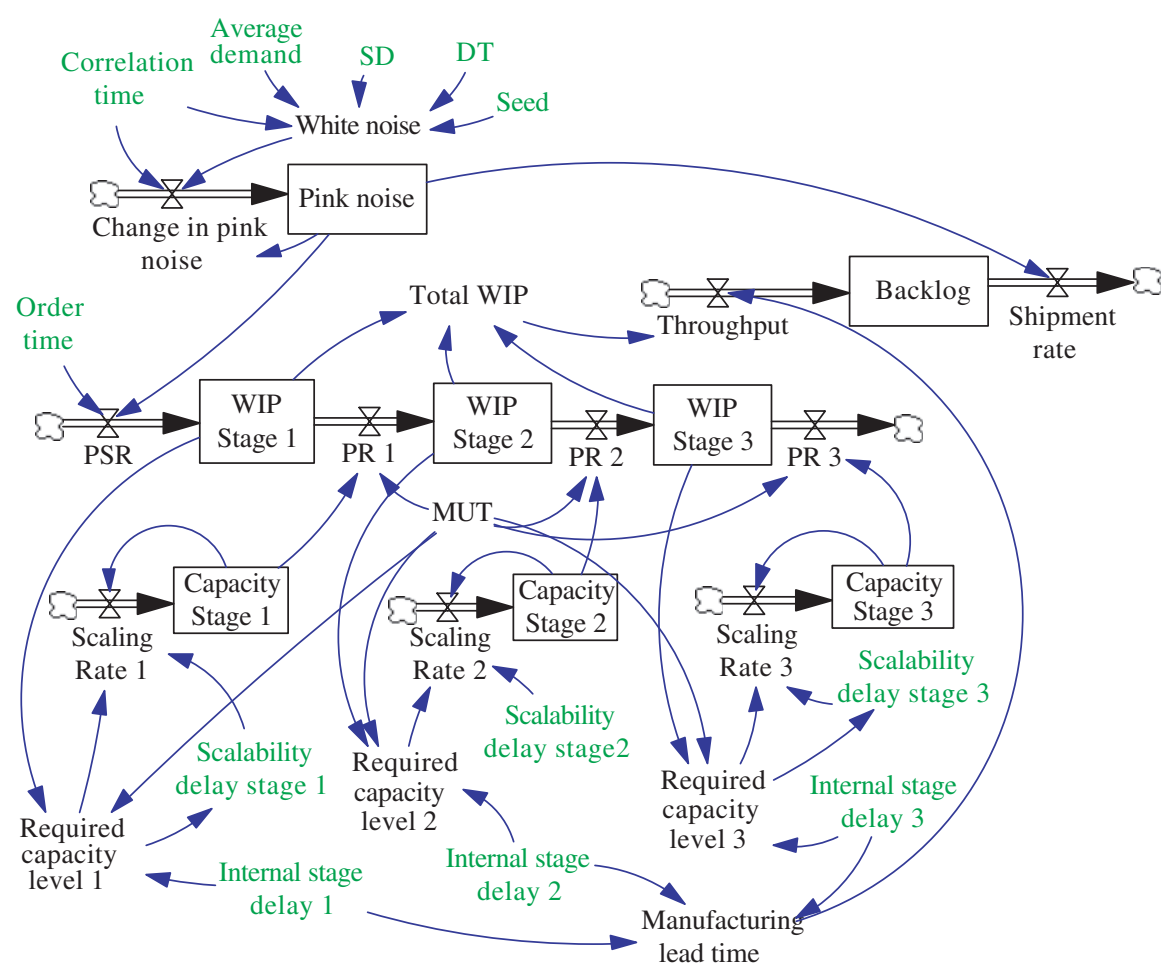

Figure 1. Dynamic capacity model in multi-stage production. 
the three-stage serial production system. It is important to note that a continuous-time model is used because it provides an acceptable approximation of the continuous dynamic capacity scalability process at that level of abstraction. Both the operations management and the SD literature support the use of continuous models for capacity planning (e.g. Holt et al. 1960, Sethi and Thompson 2000, Anderson et al. 2005). Finally, similar dynamic characteristics can be obtained using discrete-time models (John et al. 1994).

\subsection{Multi-stage production system}

A manufacturing system in which several production activities have been functionally aggregated into different production stages is considered. There are many reasons for wanting to aggregate production activities into stages. First, in most manufacturing systems (e.g. semiconductors, automotive and assembly industries) production activities are naturally grouped into well-identifiable production stages. Second, when dealing with multi-product systems, changing of setups to switch from one product to another are often performed on major sub-systems of machines (e.g. on a production line) rather than on individual machines. Controlling the production of each individual machine may, therefore, not be appropriate in such cases. Finally, having fewer points to control makes the dynamic capacity problem simpler and the implementation of a capacity management policy easier.

The multi-stage production system considered is a WIP-based control system where the WIP level is observed and controlled by varying the production rate. The production rate can vary by utilising the dynamic capacity property of these systems. In addition, the backlog of the system is monitored as a performance measure for the responsiveness of the system. The backlog is calculated based on the overall throughput of the system and its manufacturing lead-time as is explained later.

\subsection{Model notation}

Let $C_{i}(t)=$ capacity level at time $t$ at stage $i$.

$B(t)=$ the backlog level at time $t$.

$W I P_{i}(t)=$ the WIP level at time $t$ at stage $i$.

$P R_{i}(t)=$ production rate at time $t$ at stage $i$.

$\operatorname{PSR}(t)=$ the production start rate at time $t$.

$A D(t)=$ the average demand at time $t$.

$C T=$ the correlation time. This constant captures the degree of inertia (dependence) in the noise process.
$S D=$ the standard deviation for the normal demand distribution.

$D T=$ the time step.

Seed $=$ the seed for the randomly generated variates of the stochastic demand data.

$T h(t)=$ the system throughput at time $t$.

$\operatorname{Sh} R(t)=$ the shipment rate at time $t$. It is the rate of physical product leaving the system.

$T W I P(t)=$ the total WIP of the system time $t$.

$M L T=$ the manufacturing lead-time. It is the time required to process products.

$R C_{i}(t)=$ required capacity at time $t$ at stage $i$.

$S D T_{i}=$ the scalability delay time. Time required to scale the capacity at stage $i$.

$S R_{i}(t)=$ scalability rate at time $t$ at stage $i$.

$I S D_{i}=$ the internal stage $i$ delay.

$M U T=$ the manufacturing unit time.

\subsection{Model logic}

\subsubsection{Stochastic market demand}

The market demand is modelled as a stochastic parameter with dependent distribution or pink noise as referred to in the SD literature. The noise is an expression used to reflect the random variation in the data due to the stochastic nature of the process that follows a certain distribution. While statistically convenient, the independent distribution assumption of demand or white noise (as in the case of most of previous dynamic capacity analysis) does not hold for real-world cases (Sterman 2000). To have a better assessment of the impact of demand uncertainty on dynamic capacity complexity, it is necessary to model demand forecast as a process with memory in which the next value of demand does not depend on the last demand but rather on the history of previous forecasts.

The demand in this model is assumed to have a continuous cumulative normal distribution function. Huh et al. (2006) state that the demand should have a continuous distribution because demand is inherently continuous; the variance in demand is often high and finally because continuous demand distribution may generate a more robust capacity plan than finite number of discrete scenarios. Equation (1) formulates the demand as white noise with a normal distribution.

$$
\begin{aligned}
\text { White Noise }(t)= & A D(t)+\left[S D^{2} * \frac{(2-(D T / C T))}{(D T / C T)}\right. \\
& * \operatorname{Normal}(0,1, \text { Seed }) . \psi
\end{aligned}
$$

Equations (2) and (3) display the values for the demand pink noise and the change in demand pink noise, respectively. 
Pink Noise $(t)=$ Change in Pink Noise Pink Noise ${ }_{0}$

$(2) \leftarrow$

Change in Pink Noise $=\frac{\text { Pink Noise }(t)-\text { White Noise }(t)}{\text { CT }} \overleftarrow{\psi}$

$(3) \leftarrow$

\subsubsection{Dynamic capacity planning and control}

Capacity scaling decisions at each production stage $(i)$ are controlled through the scaling rate in Equation (4).

$$
C_{i}(t)=\operatorname{SR}_{i}^{*}(t) \leftarrow C_{i} \stackrel{\cdot \psi}{1}(t) . \psi
$$

The equation for the scaling rate at each stage is determined by the required capacity together with the scalability delay time (SDT) (Equation (5)).

$$
S R_{i}(t)=\frac{C_{i}(t)-R C_{i}(t)}{S D T_{i}} . \overleftarrow{\psi}
$$

The SDT in this model is a varying parameter, which is function of the type of capacity to be scaled (Equation (6)). This is an important assumption to capture the real industrial production scenarios since the time to add a spindle to a machine, for example, is indeed less than that required to add a machine to an existing line. Classical capacity scalability work had either a simple assumption of instantaneous scalability or a fixed time for scaling system's capacity.

$$
S D T_{i}=\mathrm{F}\left\{X_{i}\right\} \leftarrow
$$

where $X_{i}$ is the type of capacity

to be scaled at stage $i . \psi$

The required capacity (Equation (7)) is calculated based on the WIP level since this is a WIP-based controlled system as explained earlier.

$$
R C_{i}(t)=\left(\frac{W I P_{i}(t)}{I S D_{i}}\right) * M U T . \psi
$$

The internal stage delay (ISD), sometimes referred to as production lead-time, is in general difficult to calculate (Hoyt 1980) because of the different sources of variability within production systems (Schmitz et al. 2002). Thus the typical assumption of a deterministic value for such a parameter is highly questionable. In this model, a stochastic variable function is used to calculate the ISD (Equation (8)). The ISD function depends on the different processes and activities in each of the system production stages.

$$
I S D_{i}=\operatorname{Random} f(\min , \max , \mu, \sigma, s) \leftarrow
$$

where 'min' is the minimum value that the probabilistic function will return, 'max' is the maximum value that will be returned, $\mu \psi$ is the mean of the random distribution, $\sigma$ fis the standard deviation of the distribution and $s$ is the seed for the randomly generated numbers of the probability distribution.

\subsubsection{Production control}

The WIP level at each stage is determined by the difference between the production rate of the current stage and the production rate of the next one (Equation (9)).

$$
\operatorname{WiP}_{i}(t)=P R_{i}(t)-P R_{i+1}(t) . \psi
$$

The production start rate is set to be equal to the demand or the pink noise (Equation (10)). The production rate is controlled by the capacity scaling level since this is the typical case in systems with dynamic capacity (Equation (11)).

$$
\begin{gathered}
P S R(t)=\text { Pink Noise }(t) \leftarrow \\
P R_{i}(t)=\frac{C_{i}(t) \leftarrow}{M U T} \cdot \psi
\end{gathered}
$$

\subsubsection{Backlog calculation}

The backlog level is generally used as an indicator for the responsiveness level of the manufacturing system. In this model, it is defined as the difference between the shipment rate (which is assumed to be exactly equal to the demand as in Equation (1)) and throughput of the system (Equation (12)).

$$
\dot{B(t)}=\operatorname{ShR}(t)-T h(t) . \psi
$$

The throughput of the system is calculated based on Little's law as the function of the total WIP and the manufacturing lead-time (Equation (13)). It was shown that in the case of a multi-stage production, the bottleneck stage is the one that controls the throughput of the overall system (Hopp and Spearman 2002). Thus the total WIP is calculated using the maximum WIP (i.e. of the bottleneck stage) accumulated in the production stages (Equation (14)). The manufacturing lead-time is also calculated based on the maximum ISD in the system (Equation (15)).

$$
\begin{gathered}
\operatorname{Th}(t)=\frac{T W I P(t) \leftarrow}{M L T} \\
T W I P(t)=M A X\left(W I P_{i}(t)\right) \leftarrow \\
M L T=M A X\left(I S D_{i}\right) . \psi
\end{gathered}
$$


In summary the proposed model is composed of three main units: the first captures the demand as a stochastic process; the second handles the dynamic capacity decisions and incorporates the uncertainty of both ISD and scaling delay time and finally the third models the multi-stage production line and calculates the different production control parameters.

\section{Industrial application}

In this section, the application of the developed model to determine and analyse dynamic capacity complexity utilising a real industrial application is demonstrated.

\subsection{Overview of the multi-stage printed circuit board assembly line}

Figure 2 displays the three main stages of a printed circuit board (PCB) assembly system on an aggregate level. The production environment is typical of multiple-product systems with batch-type production control policy for every product.

The first stage is the surface mount automatic assembly devices responsible for mounting the small chips and components over the pads of the PCB. This stage contains four main processes which are screen printing of the PCB with solder paste, mounting the chips by the pick and place machine, solidifying the solder paste using thermal ovens and finally in-circuit testing ICT.

The second stage is responsible for the manual assembly of another type of electronic components called 'thru-hole' components. This stage is composed of multiple manual assembly stations followed by soldering process using a wave-soldering machine. A manual touch-up is the final process in this stage.

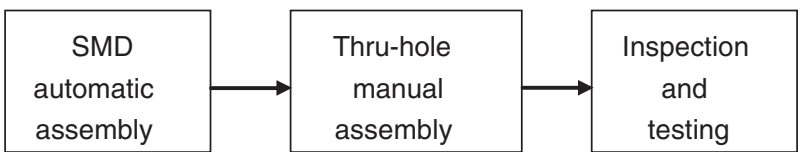

Figure 2. Stages for PCB assembly line.
The assembled PCBs go to the final stage for overall inspection and testing. The processes in this stage involve manual inspection, automatic ICT, operational testing and burn-in heavy duty testing of sampled products.

\subsection{Input data}

The product considered in this example is the computer motherboard. Two types of data are required to demonstrate the developed model, the demand model and the production system data. The demand data is shown in Table 1 while the production system data is shown in Table 2. Figure 3 shows the demand generated from the data given in Table 1.

\subsection{Numerical simulation results}

In this section, the results of various simulation experiments conducted to investigate the impact of the sources of operational complexity on the dynamic capacity planning are reported. In this analysis, the scaling rate is used as the main performance measure that can provide insight into the complexity of the dynamic capacity planning problem in terms of effort and cost. In addition, backlog and throughput are also used as performance measures in some of the conducted analysis to evaluate responsiveness and efficiency of the developed capacity planning system.

\subsubsection{Comparing stochastic and deterministic analysis}

The first analysis compares the cases where the three main sources of complexity in the developed dynamic capacity system are modelled using stochastic and deterministic data. Stochastic data means data with probabilistic representation (i.e. a mean and standard deviation), and deterministic data is represented by a constant that is equal to the mean value of the probabilistic variables. The main objective of such comparison is to highlight the impact of these sources

Table 1. Data for demand.

\begin{tabular}{|c|c|c|}
\hline Data & Value & Comments \\
\hline Average demand (AD) & 20,000 boards/week & This is the value of the average batch per customer order. \\
\hline Standard deviation (SD) & \pm 5000 boards/week & This reflects a high degree of marketing fluctuation. \\
\hline Correlation time $(\mathrm{CT})$ & 4 weeks & $\begin{array}{l}\text { This means that each demand forecast depends on the actual } \\
\text { data of the preceding month. }\end{array}$ \\
\hline Time step (DT) & 0.125 week & \\
\hline Seed & 10 & $\begin{array}{l}\text { Used to generate random variates for the normally distributed } \\
\text { demand data. }\end{array}$ \\
\hline
\end{tabular}


Table 2. Data for the three production stages of PCB assembly line.

\begin{tabular}{|c|c|c|}
\hline & ISD (weeks) & SDT (weeks) \\
\hline Stage 1 & Random uniform $(0.9,1.1,0)$ & If-then else (required capacity level $1>2500,1,0.5$ ) \\
\hline Comments & $\begin{array}{l}\text { The ISD varies randomly between } 0.9 \text { and } \\
1.1 \text { weeks with a uniform distribution. This } \\
\text { stage has the shortest delay due to its } \\
\text { automatic nature. The variation sources are } \\
\text { the variability in the processing times of the } \\
\text { different machines in this stage. }\end{array}$ & $\begin{array}{l}\text { If the required capacity to be scaled is below } 2500 \text { boards/ } \\
\text { week, then the pick and place } m / c \text { is reconfigured by } \\
\text { adding more feeders. This requires } 0.5 \text { week for } \\
\text { installation, reprogramming and ramp up. If the required } \\
\text { capacity to be scaled is above } 2500 \text { boards/week, then } \\
\text { another pick and place } m / c \text { is added to the line. This } \\
\text { requires } 1 \text { week for installation, calibration and ramp up. }\end{array}$ \\
\hline Stage 2 & Random uniform $(1.3,1.6,0)$ & 0.5 \\
\hline Comments & $\begin{array}{l}\text { The variation is larger than previous stage due } \\
\text { to many labour involved with different } \\
\text { learning curves. This is also the reason for } \\
\text { this stage being with the longest delay. }\end{array}$ & $\begin{array}{l}\text { The scaling in this stage is based on hiring more workers or } \\
\text { adding extra shifts. The delay is due to different } \\
\text { administrative and training procedures involved. }\end{array}$ \\
\hline Stage 3 & Random uniform $(1,1.3,0)$ & If-then else (required capacity level $3>10,000,0.7,0.5$ ) \\
\hline Comments & $\begin{array}{l}\text { The variations in this stage are due to the } \\
\text { variability of both the testing stations } \\
\text { machines and the labour involvement in this } \\
\text { stage. }\end{array}$ & $\begin{array}{l}\text { The scalability options here are either hiring more workers } \\
\text { (delay is } 0.5 \text { weeks) if the required capacity is less than } \\
10 \mathrm{~K} \text { boards/week or increasing the test stations (delay is } \\
1 \text { week) if the required capacity is more than } \\
10 \mathrm{~K} \text { boards/week. }\end{array}$ \\
\hline
\end{tabular}

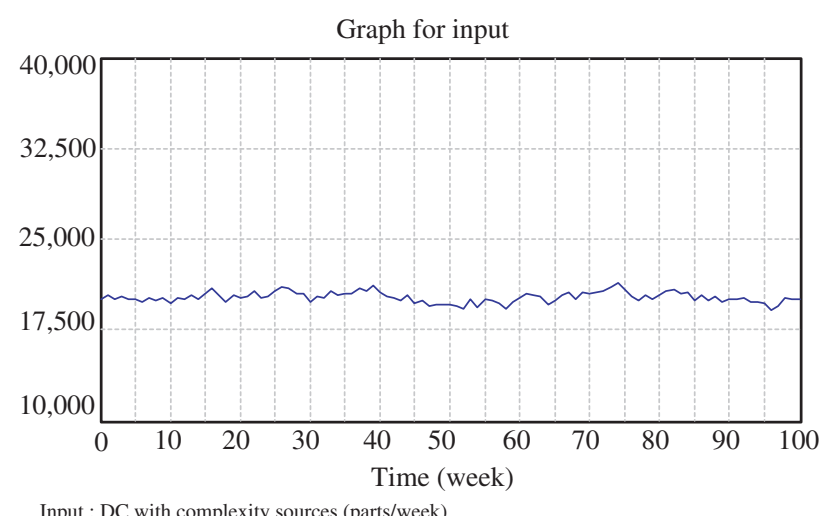

Input : DC with complexity sources (parts/week)

Figure 3. Input demand.

on the complexity of the dynamic capacity planning. Figure 4 (a)-(c) compares the scaling rate, as a performance measure of capacity scalability, in the stochastic case (left side) with that in the deterministic case (right side) at each of the three production stages in the PCB line.

Analysis of Figure 4 reveals the following observations (DC refers to Dynamic Capacity):

- The levels of the capacity scaling rate in each stage for the two cases illustrate the effect of the various sources of complexity. The magnitude of the scaling rates in the stochastic case has much higher values than those of the deterministic case (roughly about four times higher). The stochastic case exhibits more oscillations (i.e. more frequent capacity changes with its associated costs) compared with the deterministic case, and the latter even reaches stability at the value of zero after some weeks. Thus incorporating the sources of complexity into capacity planning increases the operational complexity of the scaling decisions in terms of their number and frequency.

- The desirable dynamic behaviour of the deterministic case compared with the stochastic case points to a fundamental trade-off decision in dynamic capacity planning. The planner has to balance the need for accurate representation of the scaling process against the desire to keep an acceptable level of controllability of that process.

- The results also show the occurrence of the 'bullwhip' effect, which is the variance in the processing rate and hence the next stage demand being greater than that of input tasks (Frank et al. 2000), in the stochastic case. This adds another dimension to the complexity of the decision regarding the level of aggregation when designing dynamic capacity planning systems.

\subsubsection{Impact of operational complexity sources on production systems' performance and responsiveness}

The throughput is a fundamental performance measure of a production system (Hopp and Spearman 2000). 
(a)

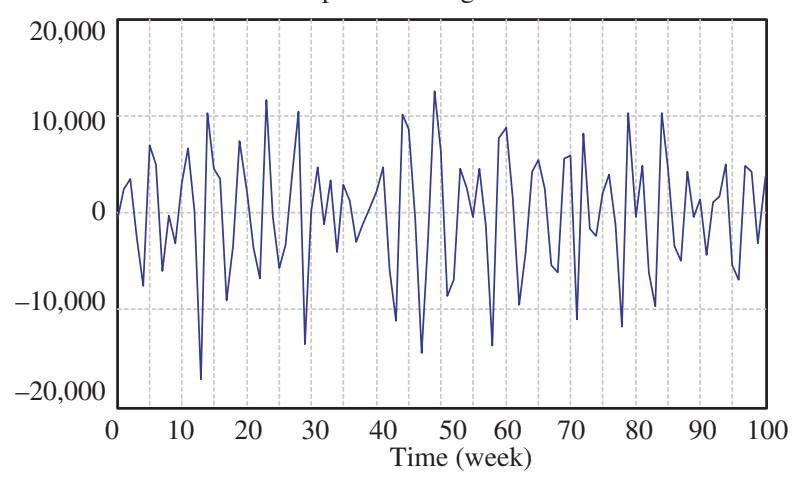

Scaling rate $1:$ DC with complexity sources (parts/week)

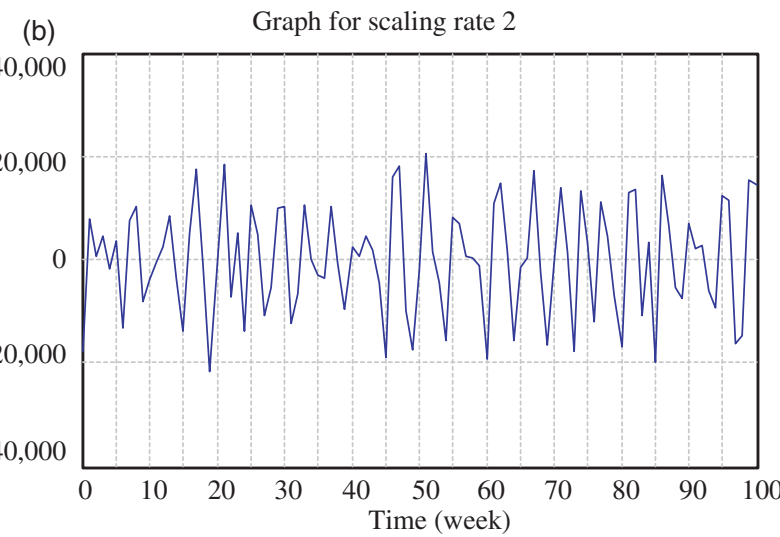

Scaling rate 2 : DC with complexity sources (parts/week)

(c)

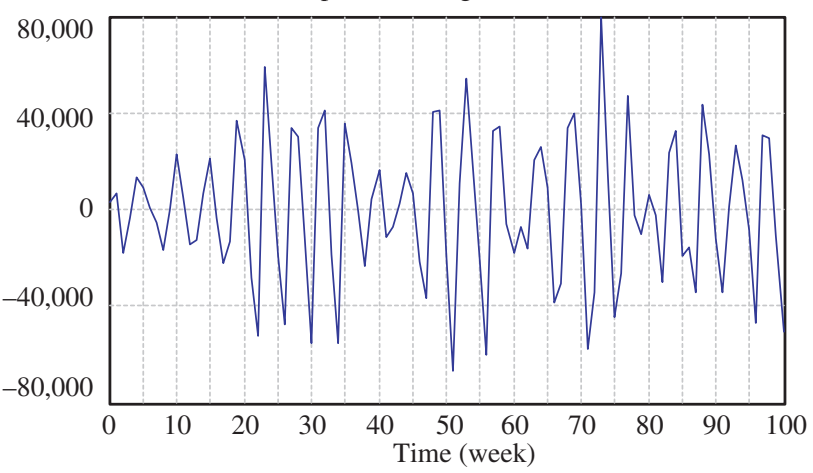

Scaling rate $3:$ DC with complexity sources (parts/week)

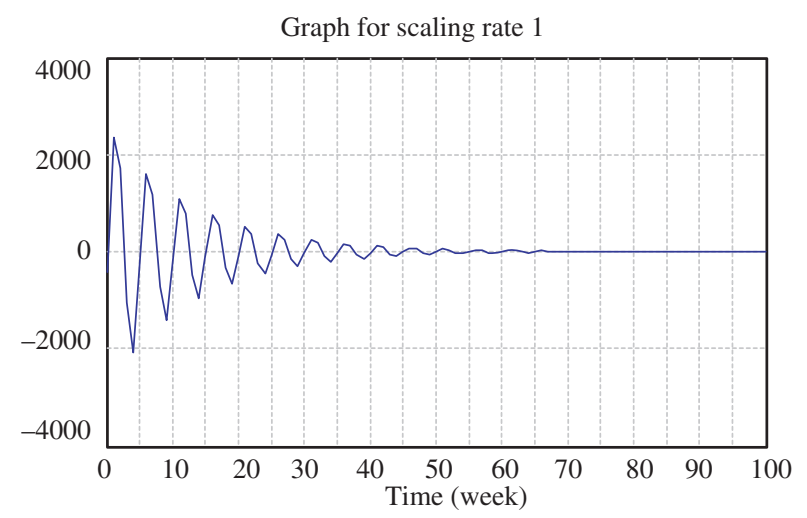

Scaling rate $1:$ DC with no complexity sources (parts/week)

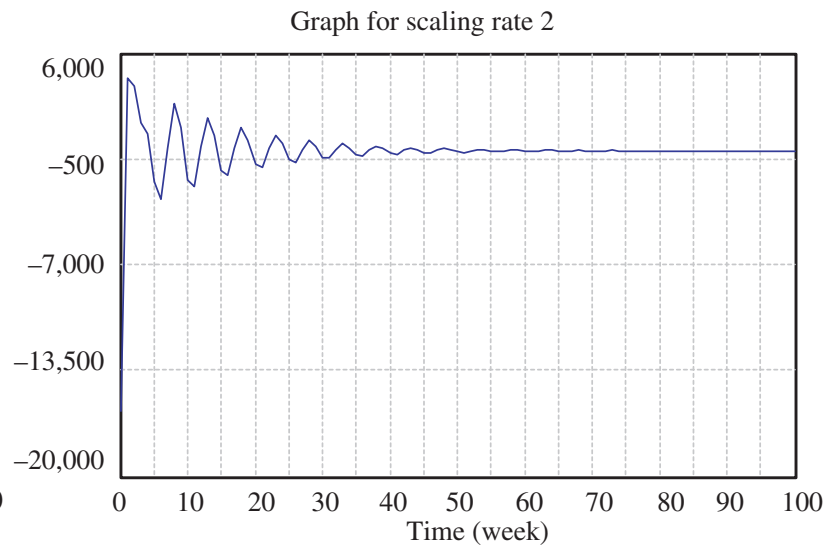

Scaling rate 2 : DC with no complexity sources (parts/week)

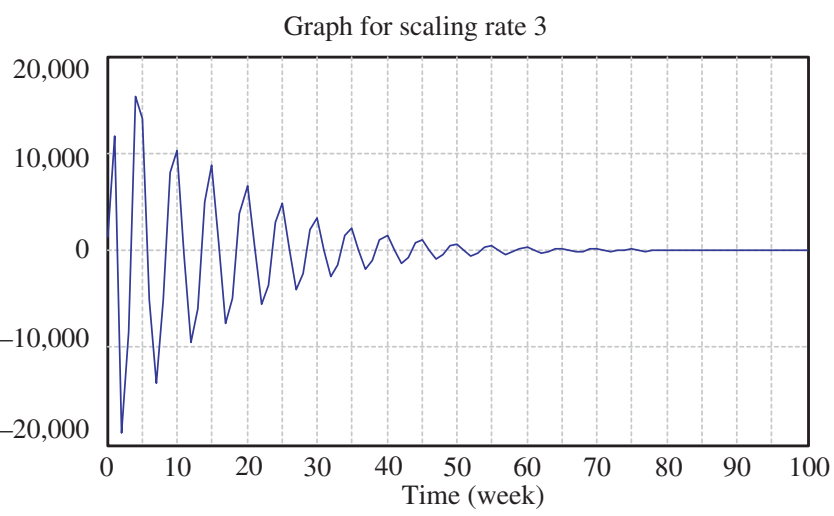

Scaling rate $3:$ DC with no complexity sources (parts/week)

Figure 4. Scalability rate in cases of stochastic analysis (left side) and deterministic analysis (right side). (a) First stage, (b) second stage and (c) third stage.

Figure 5 compares the evolution of throughput of the developed multi-stage production system in (a) stochastic case and (b) deterministic case.

The result shows that the variations in the sources of complexity negatively affect the performance of the system in comparison with the case where these variations are eliminated. The uncertainty associated with these sources led to higher than the required level of throughput in addition to dynamic oscillations (changes) that will affect the stability of the system. This leaves the capacity planner with another trade-off decision to compromise between efficient production 
(a)

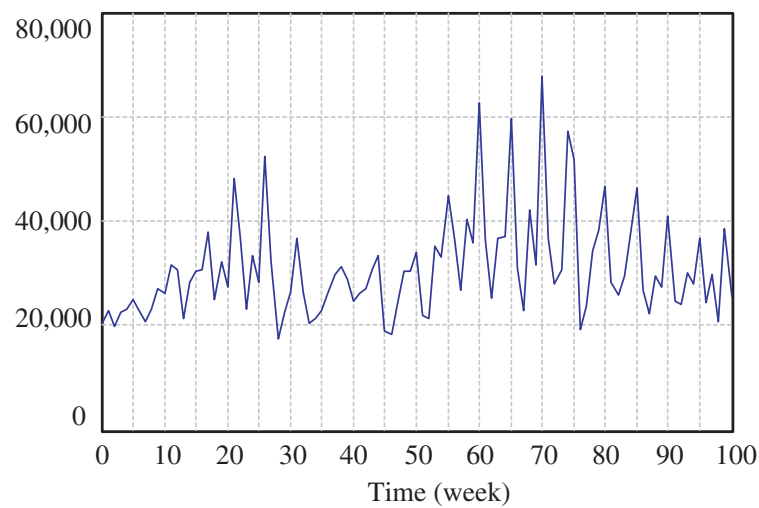

Throughput : DC with complexity sources (parts/week) (b) Graph for throughput

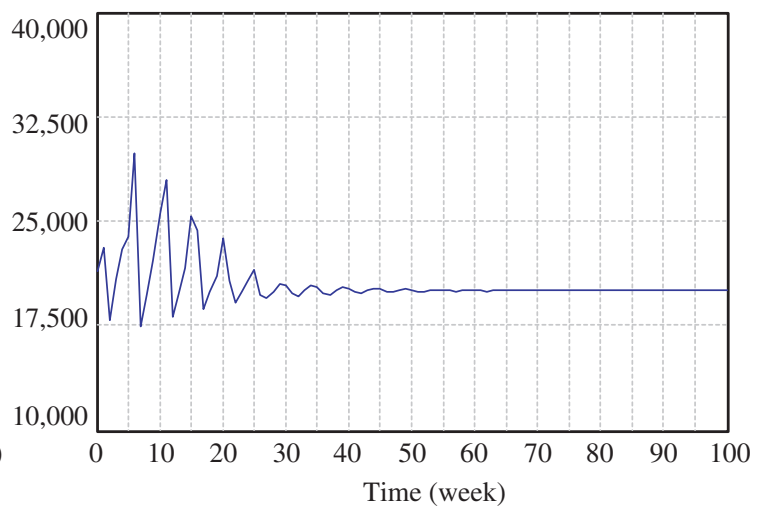

Throughput : DC with no complexity sources (parts/week)

Figure 5. Throughput level. (a) Stochastic case and (b) deterministic case.

(a)

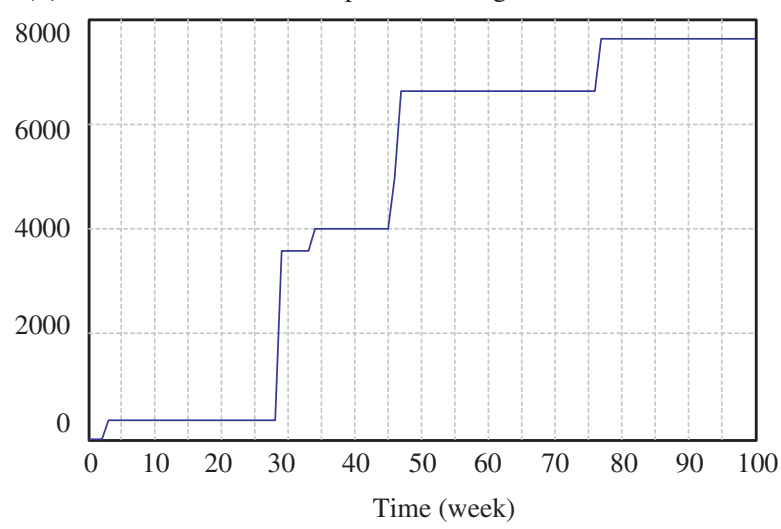

Backlog : DC with complexity sources (parts/week)

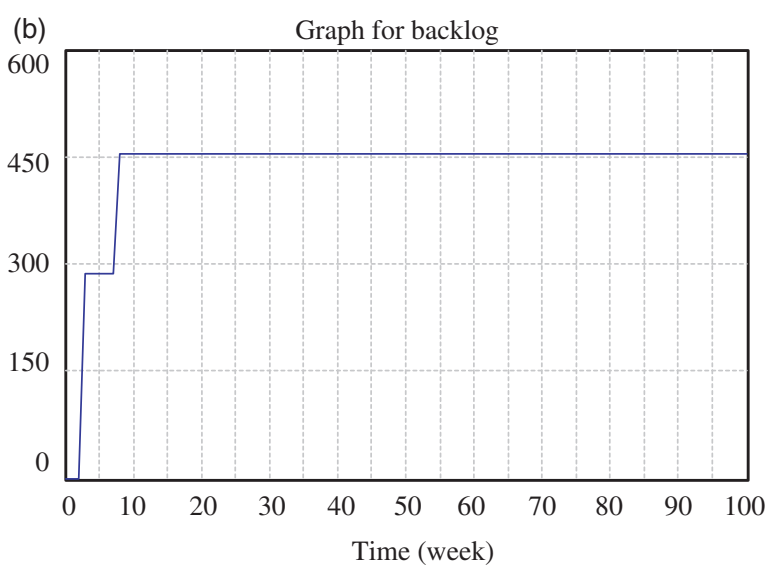

Backlog : DC with no complexity sources (parts/week)

Figure 6. Backlog level. (a) Stochastic case and (b) deterministic case.

in terms of cost (less inventory and oscillation) and at the same time a realistic abstraction and representation of the sources of operational complexity.

Backlog is also a crucial indicator for the degree of responsiveness especially in systems employing dynamic capacities to maintain a short market leadtime. Figure 6 shows the backlog level in both cases of stochastic and of deterministic analysis of the developed dynamic capacity model.

Results in Figure 6 highlight that, in general, the backlog level for the deterministic case is much lower than the stochastic case although at the first 30 weeks, the situation was reversed. This indicates that ignoring the uncertain nature of the complexity sources can lead to false assessment of the level of responsiveness of the production system. In other words, having 450 (about $2 \%$ of the demand) boards only as a backlog indeed means a very high level of responsiveness; however, realistically speaking the variation in the complexity sources will lead to an average backlog of $4 \mathrm{~K}$ boards (approximately $20 \%$ ) which is a much lower responsiveness level.

\subsubsection{Comparative assessment for sources of operational complexity in dynamic capacity planning systems}

In this section, the impact of each of the three sources of operational complexity considered in this study on the dynamic capacity planning is discussed. The used performance measure is the scaling rate as an indicator of the required capacity planning effort and cost. The analysis is based on fixing two of the three sources of uncertainty, where a deterministic value of their average is used, while observing the impact of the third source over scaling rate.

Figure 7 (a)-(c) shows the impact of each of the three considered sources of operational complexity 
(a)

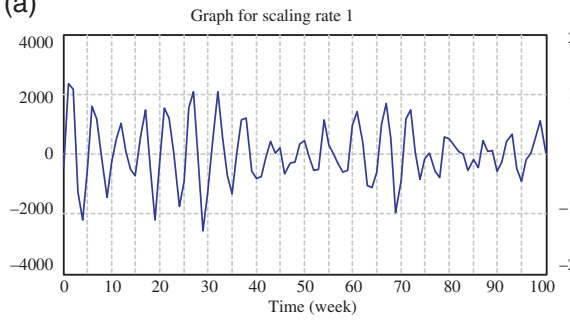

(b) Scaling rate $1:$ Effect of demand on DC complexity (parts/week)

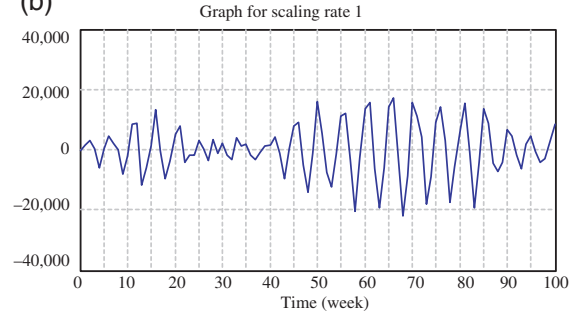

(c)

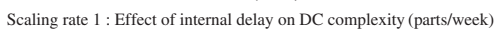

Graph for scaling rate 1

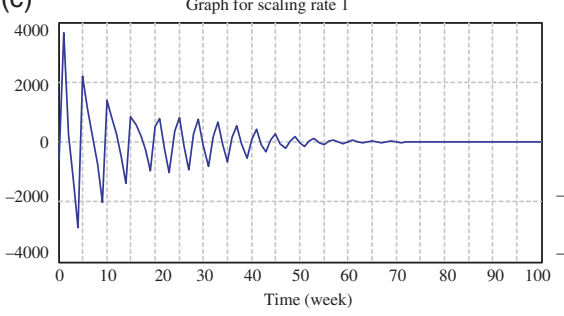

Graph for scaling rate 2

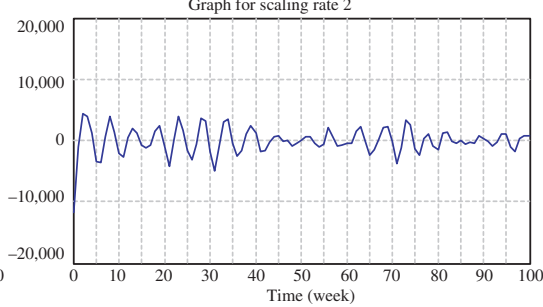

Scaling rate 2 : Effect of demand on DC complexity (parts/week)

Graph for scaling rate 2

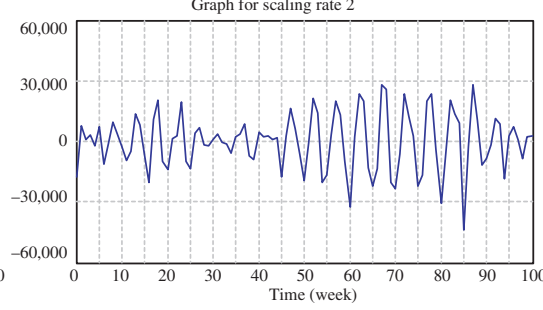

Scaling rate 2 : Effect of internal delay on DC complexity (parts/week)

Graph for scaling rate 2

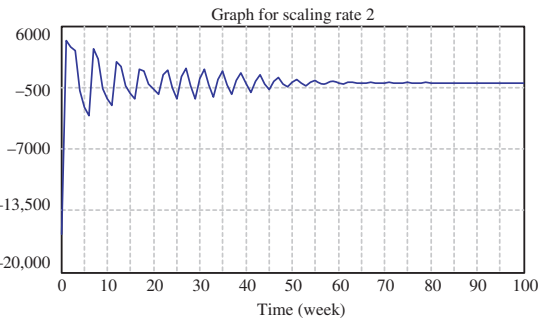

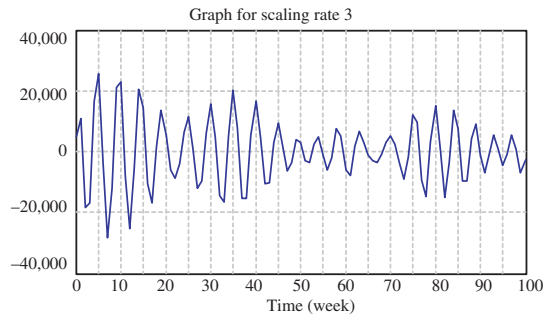

Scaling rate 3 : Effect of demand on DC complexity (parts/week) Graph for scaling rate 3

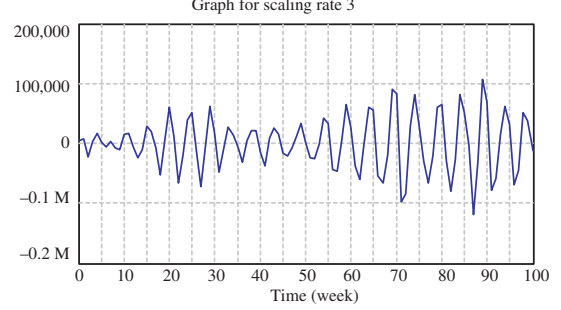

Scaling rate 3 : Effect of internal delay on DC complexity (parts/week) Graph for scaling rate 3

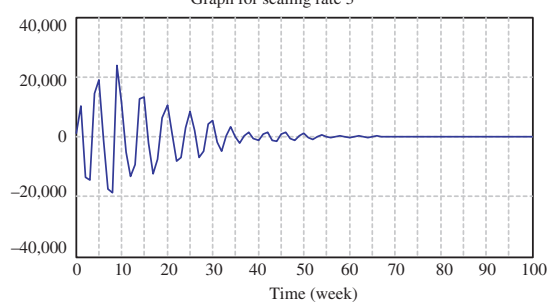

Figure 7. Impact of each of the three considered sources of operational complexity over the scaling rate at each of the three stages of production. (a) Stochastic demand, (b) internal stage delay and (c) scalability delay time.

over the scaling rate at each of the three stages of production.

The following three main observations can be deduced based on the obtained results:

- The ISD, in a multi-stage production system, is the main source of operational complexity in the capacity scalability process. This is demonstrated through having the greatest number of oscillations for the scaling rate across the three production stages. In addition, the scaling rate has the highest value at each stage with the ISD. It is important to note that the scaling rate experiences many oscillations (i.e. more frequent capacity changes and its associated costs) due to the stochastic demand, which makes it the second source for the operational complexity in the capacity scalability process.

- An interesting observation is that the scalability delay time, based on the magnitude and number of oscillations, has a minimal contribution to the operational complexity as the scaling rate tends to reach zero after a period of time. This is because after a period of time, and since demand is assumed to be stable, the production (after some scaling of capacity) will be able to exactly match the demand and thus no further capacity scaling is required. This indicates that the share of the SDT in the operational complexity of the scaling process is proportional to the stability of the market demand.

- The 'bullwhip' effect is clear in the impact of the three operational complexity sources over the scaling rate across the production stages. This suggests that bullwhip is another source of operational complexity in the capacity scalability process and highlights the importance of studying the conditions under which this phenomenon occurs to better manage the complexity of this process.

\section{Conclusions and recommendations}

This study of the dynamic capacity scalability in multistage production systems is focused, for the first time, on the intuitive understanding of the operational complexity associated with the capacity scaling process in these systems. The operational complexity 
was defined as the required effort measured in terms of magnitude and frequency of capacity scaling in response to dynamic demand. An approach based on SD was presented to model the dynamic nature of this scalability process.

The unique feature of this modelling approach is its ability to identify and quantify the three main sources of the operational complexity relevant to this problem. These are the stochastic demand, ISD and capacity scaling delay time. The proposed approach was illustrated by a case study for a typical industrial multi-stage PCB assembly line. Several results were demonstrated using numerical simulation, leading to the following conclusions:

(1) The uncertainties associated with the considered sources of complexity were quantitatively proven to increase the level of operational complexity of the dynamic capacity planning problem. This was demonstrated by the magnitude and frequency of the scaled capacity in response to the varying demand.

(2) A trade-off between the complexity and controllability of the capacity scalability must be exercised by the capacity planner. A desirable high level of controllability requires capturing the stochastic characteristics of the uncertainty sources of capacity scalability, which would lead to increasing the operational complexity of the planning decisions.

(3) The performance of the production system in terms of its throughput and responsiveness was negatively affected by the considered sources of operational complexity. This is critical particularly in those systems that adopt dynamic capacity to hedge against dynamic demand.

The reported results together with the previous conclusions lead to the following recommendations for better managing the operational complexity in a dynamic capacity planning environment in multistage, multi-product production systems:

(1) Reducing randomness through better information handling or tighter control is essential to decrease the degree of uncertainty associated with forecasting the demand, the manufacturing lead-time and scalability delay time.

(2) More effort should be devoted to stabilising and/or accurate calculation of the ISD. The presented results showed that it has the highest impact on the operational complexity level.

(3) The conditions under which the bullwhip effect occurs should be determined and used as constraints for scalability decisions. Results showed that the bullwhip effect contributes to the operational complexity of dynamic capacity planning in multi-stage production systems.

This work is one of the first attempts to study some of the sources and effects of operational complexity in dynamic capacity planning problem; however, further work is required to investigate the effect of other sources of uncertainty on its complexity. Conducting designed experiments to understand the different interactions between the systems' parameters would be a logical extension. In addition, studying the relationship between the model parameters and the structural and operational complexity relationships can lead to the development of a general framework for optimal capacity management in dynamic manufacturing in changeable environments.

Finally, uncertainty cannot be avoided in today's changeable manufacturing environment; however, a proper understanding of the sources of this uncertainty helps the capacity planner to better manage the scalability process.

\section{Acknowledgements}

The contributions from the Canada Research Chairs (CRC) program and the Natural Sciences and Engineering Research Council (NSERC) of Canada in support of this research are greatly acknowledged.

\section{Notes on contributors}

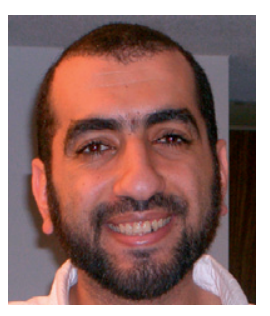

Dr Ahmed Deif received his $\mathrm{PhD}$ and MASc in Industrial and Manufacturing Systems Engineering from the University of Windsor, Canada in 2006 and 2003, respectively. He received his $\mathrm{BSc}$ from The American University in Cairo, Egypt (1999). He currently works as Assistant Professor at the Industrial Systems Engineering, University of Regina. His research interests include manufacturing capacity management, manufacturing planning and control and dynamic analysis of manufacturing systems. Dr Deif is a Senior Member of the Society of Manufacturing Engineers (SME), Institute of Industrial Engineers (IIE) and a Research Affiliate of the International Academy for Production Engineering (CIRP).

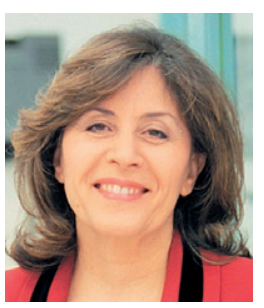

Hoda A. ElMaraghy is currently Canada Research Chair in Manufacturing Systems, Professor of Industrial and Manufacturing Systems Engineering and Director of the Intelligent Manufacturing Systems (IMS) Centre at the University of Windsor. She is a Fellow of the Canadian Society of Mechanical Engineers (CSME), 
the Society of Manufacturing Engineers (SME) and the International Academy for Production Engineering (CIRP) and a Member of the American Society of Mechanical Engineers (ASME). Her teaching and research interests include manufacturing systems design, modelling, flexibility, reconfiguration, control, quality, inspection, assembly, process planning and robotics. Dr Hoda ElMaraghy has published more than 350 papers in scientific and professional journals and conferences, edited 3 books and contributed to 13 other books. She is a Member of Editorial Boards of four International Scientific and Engineering Journals. She received a Mechanical Engineering Bachelor degree from Cairo University, Egypt and Master's and PhD degrees from McMaster University, Canada where she became Professor and founding Director of the Flexible Manufacturing Systems Centre until joining the University of Windsor as the Dean of Engineering in 1994.

\section{References}

Anderson, E., Morrice, D., and Lundeen, G., 2005. The 'physics' of capacity and backlog management in service and custom manufacturing supply chains. System Dynamics Review, 22 (3), 217-247.

Asl, R. and Ulsoy, A., 2002. Capacity management via feedback control in reconfigurable manufacturing systems. In: Proceedings of Japan-USA symposium on flexible manufacturing automation, Hiroshima, Japan.

Deif, A. and ElMaraghy, W., 2006. A control approach to explore capacity scalability scheduling in reconfigurable manufacturing systems. Journal of Manufacturing Systems, 25 (1), 12-24.

Deif, A. and ElMaraghy, W., 2007a. Integrating static and dynamic analysis in studying capacity scalability in RMS. International Journal of Manufacturing Research, 2 (4), 414-427.

Deif, A. and ElMaraghy, H., 2007b. Assessing capacity scalability policies in RMS using system dynamics. International Journal of Flexible Manufacturing System, Special Issue on Dynamic capacity, 19, 128-150.

Deshmukh, A., Talavage, J., and Barash, M., 1998. Complexity in manufacturing systems, part 1: Analysis of static complexity. IIE Transactions, 30, 645-655.

Duffie, N. and Falu, I., 2002. Control-theoretic analysis of a closed loop PPC system. Annals of CIRP, 52 (1), 379-382.

ElMaraghy, H.A., 2006. A complexity code for manufacturing systems. In: 2006 ASME international conference on manufacturing science \& engineering (MSEC), Symposium on advances in process \& system planning, Ypsilanti, MI, USA.

ElMaraghy, H.A., Kuzgunkaya, O., and Urbanic, J., 2005. Comparison of manufacturing system configurations A complexity approach. 55th CIRP Annals, 54 (1), 445-450.

Evans, G. and Naim, M., 1994. The dynamics of capacity constrained supply chains. In: Proceedings of international system dynamics conference, Stirling, Scotland, 28-35.
Forrester, J.W., 1961. Industrial dynamics. Cambridge, USA: MIT Press.

Frank, C., et al., 2000. Quantifying the bullwhip effect: the impact of forecasting, lead-time and information. Management Science, 46 (3), 436-443.

Frizelle, G., 1998. The management of complexity in manufacturing. London: Business Intelligence.

Goncalves, P., Hines, J., and Sterman, J., 2005. The impact of endogenous demand on push-pull production systems. System Dynamics Review, 22 (3), 217-247.

Helo, P., 2000. Dynamic modeling of surge effect and capacity limitation in supply chains. International Journal of Production Research, 38 (17), 4521-4533.

Holt, C.C., Modigliani, F, Muth, J.F., and Simon, H.A., 1960. Planning production, inventories, and work force. Englewood Cliffs, NJ: Prentice-Hall.

Hopp, W. and Spearman, M., 2000. Factory physics. New York: McGraw Hill.

Hoyt, J., 1980. Determining lead time for manufactured parts in a job shop, In: J.J. Pennsanken, ed. Computers in manufacturing. Florida, USA: Auerbach Publishers, $1-12$.

Huh, WT, Roundy, RO, and Cakanyildirim, M., 2006. A general strategic capacity planning model under demand uncertainty. Naval Research Logistics, 2, 137-150.

John, S., Naim, M., and Towill, DR., 1994. Dynamic analysis of a WIP compensated support system. International Journal of Manufacturing System Design, 1 (4), 283-297.

Kim, J.-H. and Duffie, N., 2004. Backlog control design for a closed loop PPC system. Annals of CIRP, 54 (1), 456-459.

Kim, J.-H. and Duffie, N., 2005. Design and analysis for a closed loop capacity control of a multi workstation production system. Annals of CIRP, 55 (1), 470-474.

Luss, H., 1982. Operation research and capacity expansion problems: A survey. Operation Research, 3 (5), 907-947.

Manne, A.S., 1967. Investments for capacity expansion, size, location, and time-phasing. Cambridge, MA: MIT Press.

Nyhuis, P., 1994. Logistic operating curves - A comprehensive method for rating logistic potentials. EURO XIII/ OR36. University of Strathclyde Glasgow.

Schmitz, J.P.M, van Beek, D., and Rooda, J., 2002. Chaos in discrete production systems. Journal of Manufacturing Systems, 21, 23-35.

Sethi, S.P. and Thompson, G.L., 2000. Optimal control theory: Applications to management science and economics. Boston, MA: Kluwer.

Sterman, J.D., 2000. Business dynamic - Systems thinking and modeling for a complex world. Boston, MA: McGraw-Hill.

Van Mieghem, J., 2003. Capacity management, investment and hedging: Review and recent developments. Manufacturing and service operation management, 5 (4), 269-302.

Vlachos, D., Georgiadis, P., and Iakovou, E., 2007. A system dynamics model for dynamic capacity planning of 
remanufacturing in closed-loop supply chains. Journal of Computers and Operation Research, 34, 367-394.

Wiendahl, H.P. and Breithaupt, J., 1999. Modeling and controlling of dynamics of production system. Journal of Production Planning \& Control, 10 (4), 389-401.

Wiendahl, H.P. and Breithaupt, J., 2000. Automatic production control applying control theory. International Journal of Production Economics, 63, 33-46.
Wiendahl, H.-P, et al., 2007. Changeable manufacturing: Classification, design, operation. Keynote Paper. CIRP Annals, 56 (2), 456-459.

Wikner, J., Naim, M., and Rudberg, M., 2007. Exploiting the order book for mass customized manufacturing control systems with capacity constrains. IEEE Transaction on Engineering Management, 54 (1), 145-155. 